\title{
Synchronization and Chaos of Unbalanced Rotors on a Flexible Structure *
}

\author{
Takuo NAGAMINE **, Yuichi SATO ** and Kosuke KAWASE ** \\ ** Graduate School of Engineering and Science, Saitama University, \\ 255, Shimo-Okubo, Sakura-ku, Saitama-shi, Saitama 338-8570, Japan \\ E-mail: nagamine@mech.saitama-u.ac.jp
}

\begin{abstract}
This paper describes the numerical calculations on the vibrations of a structure excited by two unbalanced rotors. When two rotors rotate on a base plate, which is free to vibrate horizontally, some kinds of vibration modes, i.e., in-phase synchronization, out-of-phase synchronization, and non-synchronization are observed. According to our investigation of rotor unbalance, we observed two types of non-synchronization. One is quasi-periodic vibration, and the other is chaos.
\end{abstract}

Key words: Unsteady State Vibration, Unbalance, Poincare Map, Limited Power Supply, Synchronization, Quasi-Periodic Vibration, Chaos

\section{Introduction}

It is important problem that rotors can safely pass through a critical speed of a structure. Thus far, the characteristics of motion of flexible rotors passing through a critical speed are investigated. $^{(1) \sim(8)}$ These studies were clarified that the relation between the driving torque required for passing the critical speed and the load torque generated by vibration. In their studies, only one rotor was used. About more than two rotors, the synchronization of rigid rotors and structure are also investigated for the application of vibration, and clarified the synchronization conditions and characteristics of the motions by averaging methods. ${ }^{(9)}(12)$ However, transient vibration has not been examined sufficiently. The aim of our research is to prevent the large vibration that occurs when rotors are synchronized. ${ }^{(13)}$ In this study, we examined the effects of the unbalance of the rotors and the interaction between rigid rotors and a support structure when the rotors pass through the critical speed of the support structure, and clarified the rotor behavior near the critical speed.

This paper describes the numerical calculations of the vibrations of the structure excited by unbalanced rotors. The calculated results also shows Lyapunov exponents of the system. When rotor speeds cannot exceed the critical speed, the rotational speeds of rotors are entrained into a single speed just below the critical speed and the phases of unbalanced rotors are locked so as to excite the structure violently. On the other hand, when two rotors operated above the critical speed, unbalance forces cancel each other, that is, vibration is very small. In our experiment, some kinds of vibration modes, i.e., in-phase synchronization, out-of-phase synchronization and non-synchronization are observed. According to our investigation of effects of the unbalanced mass, we observed two types of non-synchronization. One is quasi-periodic vibration and the other is chaos. 


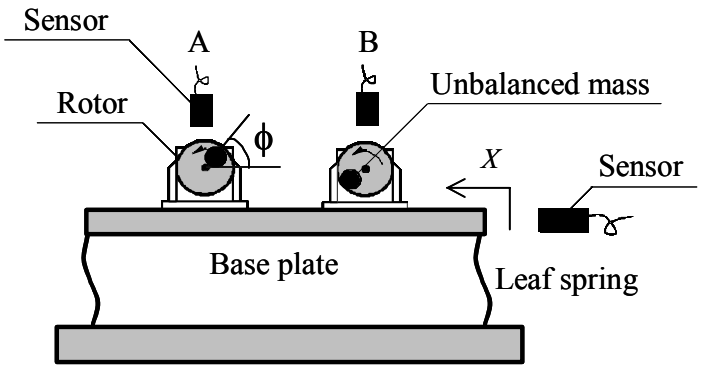

Fig.1 Analytical model

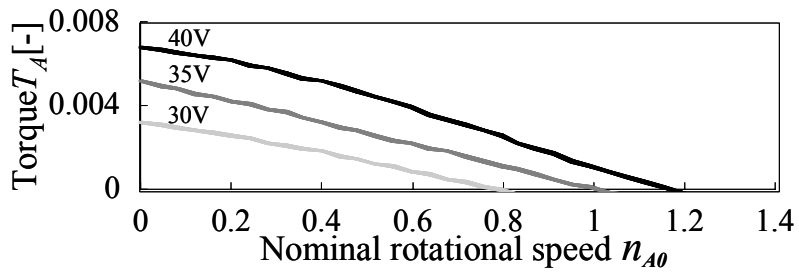

(a) Rotor $\mathrm{A}$

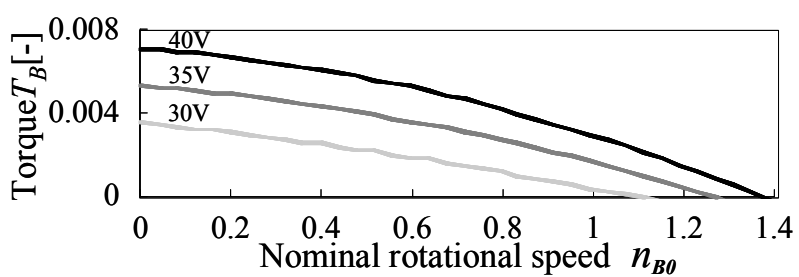

(b) Rotor B

Fig.2 Measured torque - speed characteristics

\section{Nomenclature}

M: Mass of support system

$i$ : $\quad$ Rotor $i(i=\mathrm{A}, \mathrm{B})$

$m_{i} e_{i}$ : Unbalanced momentum added to rotor $i$

$J_{i}$ : $\quad$ Polar moment of inertia of rotor $i$

$m_{i}$ : Eccentric mass of rotor $i$

$n_{i 0}=\omega_{i} / \omega_{p}$ : Nominal rotational speed (rotational speed when base plate is fixed) $n_{i 1}=\omega_{i} / \omega_{p}$ : Actual rotational speed (rotational speed when base plate is not fixed) $T_{i}=\operatorname{Trq}_{i} /\left(\Omega^{2}{ }_{i 0} m_{i} e_{i} g\right)$ : Dimensionless torque

$\operatorname{Tr}_{i}$ : Motor driving torque

$x$ : $\quad$ Displacement of base plate $X=x / e_{0}$

$\Omega_{i 0}=\omega_{n i} / \omega_{p}$

$\Omega_{i 1}=\omega_{n i} / \sqrt{g / e_{0}}$

$\phi_{i}$ : Angular displacement of rotor $i$

$$
\gamma_{i}=\frac{m_{i}}{M+\sum_{i=A}^{B} m_{i}}
$$

$\eta_{i}=e_{i} / e_{0} \quad\left(e_{0}=0.024 \mathrm{~m}\right)$

$\zeta_{i}$ : $\quad$ Damping ratio of rotor $i$

$\zeta_{p}$ : Damping ratio of base plate 
$\tau: \quad$ Dimensionless time $\left(\tau=\omega_{p} t\right)$

$\omega_{i}$ : Angular frequency of rotor $i$

$\omega_{p}: \quad$ Natural angular frequency of system $(=4.7 \times 2 \pi \mathrm{rad} / \mathrm{s})$

$\omega_{n i}=\sqrt{m_{i} e_{i} g /\left(J_{i}+m_{i} e_{i}^{2}\right)}$

\section{Equations of motion and procedure}

\subsection{Analytical model}

Figure 1 shows the analytical model. Two unbalanced rotors are fixed on a base plate, which can vibrate horizontally. The natural frequency $v_{p}$ of the base plate is $4.7 \mathrm{~Hz}$.

The dc motors are installed with their axes in parallel and rotate counterclockwise. The diameter of a rotor attached to a motor is $60 \mathrm{~mm}$. Unbalanced mass is placed on the rotor at a radial position of $25 \mathrm{~mm}$ from the center.

The motor speed is controlled by the supply voltage. Figure 2 shows the measured relation between dimensionless torque and nominal rotational speed of the supply voltage. As rotational speed increases, torque of each rotor decreases linearly. For a given supply voltage the rotor runs at speed $n_{i 0}(i=A, B)$ which is called nominal rotational speed, when the base plate is fixed. On the other hand, when the base plate is free to vibrate, the rotational speed of the rotor is influenced by the vibration of the base plate. Therefore, the rotor rotates at actual rotational speed $n_{i 1}$ less than $n_{i 0}$. We consider that rotor driving conditions expressed in terms of the nominal rotational speed are more understandable than those expressed in terms of the voltage when examining the relationship with the critical speed. Therefore, the driving conditions were expressed using the nominal rotational speed in later sections.

\subsection{Equations of motion}

The analytical model is consists of rigid rotors and rigid support bearings. we suppose that the base plate vibrates in a $x$ direction only, because vertical vibration is very small. The equations of motion for the system shown in Fig. 1 are derived as follows,

Base plate:

$$
\frac{d^{2} X}{d \tau^{2}}+2 \zeta_{p} \frac{d X}{d \tau}+X-\sum_{i=A}^{B} \gamma_{i} \eta_{i}\left\{\left(\frac{d \phi_{i}}{d \tau}\right)^{2} \cos \phi_{i}+\frac{d^{2} \phi_{i}}{d \tau^{2}} \sin \phi_{i}\right\}=0
$$

Rotors A, B:

$$
\frac{d^{2} \phi_{i}}{d \tau^{2}}+2 \zeta_{i} \Omega_{i 0} \frac{d \phi_{i}}{d \tau}+\Omega_{i 0}^{2} \cos \phi_{i}=\Omega_{i 1}^{2} \frac{d^{2} X}{d \tau^{2}} \sin \phi_{i}+T_{i} \quad, \quad(i=A, B)
$$

where $T_{i}$ is torque which is the measured values shown in Fig. 2.

\subsection{Procedure for solution}

Equations (1) and (2) are solved numerically. The data used in the numerical simulations were those obtained by measurements. We introduce the following vectors

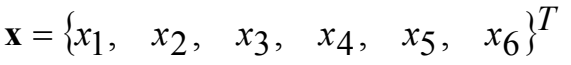

$$
\begin{aligned}
& =\left\{X, \quad X^{(1)}, \quad \phi_{A}, \phi_{A}^{(1)}, \quad \phi_{B}, \phi_{B}^{(1)}\right\}^{T} .
\end{aligned}
$$


Here notations superscripts ${ }^{(1)}$ and ${ }^{(2)}$ denote differentiation with respect to dimensionless time $\tau$.

$$
\begin{aligned}
& \left({ }^{(1)}=d / d \tau,{ }^{(2)}=d^{2} / d \tau^{2}\right) \text { and } \tau=\omega_{p} t \text {. } \\
& \mathbf{X}=\frac{d \mathbf{x}}{d \tau}=\left\{X_{1}, \quad X_{2}, \quad X_{3}, \quad X_{4}, \quad X_{5}, \quad X_{6}\right\}^{T} \\
& =\left\{x_{2}, \quad x_{2}^{(1)}, x_{4}, \quad x_{4}^{(1)}, x_{6}, x_{6}^{(1)}\right\} .
\end{aligned}
$$

It is necessary for calculation of Lyapunov exponents to treat the following equations

$$
\begin{aligned}
& \frac{d \boldsymbol{\Phi}(\tau)}{d \tau}=\mathbf{Y} \boldsymbol{\Phi}(\tau), \\
& \boldsymbol{\Phi}(0)=\mathbf{I}, \\
& \mathbf{Y}=\frac{\partial \mathbf{X}}{\partial \mathbf{x}},
\end{aligned}
$$

where $\boldsymbol{\Phi}(\tau)$ is matrix (6th order square matrix), $\mathbf{I}$ is the 6th order unit matrix, $\mathbf{Y}$ is the Jacobian matrix (6th order square matrix).

$$
\frac{d}{d \tau}\left[\begin{array}{c}
\mathbf{x} \\
\varphi_{1} \\
\varphi_{2} \\
\varphi_{3} \\
\varphi_{4} \\
\varphi_{5} \\
\varphi_{6}
\end{array}\right]=\left[\begin{array}{c}
\mathbf{X} \\
\mathbf{Y} \cdot \varphi_{1} \\
\mathbf{Y} \cdot \varphi_{2} \\
\mathbf{Y} \cdot \varphi_{3} \\
\mathbf{Y} \cdot \varphi_{4} \\
\mathbf{Y} \cdot \varphi_{5} \\
\mathbf{Y} \cdot \varphi_{6}
\end{array}\right]
$$

where $\varphi_{i}$ is the 6 th column vector of

$$
\Phi=\left[\begin{array}{llllll}
\varphi_{1} & \varphi_{2} & \varphi_{3} & \varphi_{4} & \varphi_{5} & \varphi_{6}
\end{array}\right]
$$

By applying the Runge-Kutta method to equation (8), $\mathbf{x}(T)$ and $\boldsymbol{\Phi}(T)$ are obtained. We apply the Gram-Schmidt orthonormalization to $\boldsymbol{\Phi}(T)$ by time step $\Delta \tau$. Then Lyapunov exponents $\lambda_{i}$ are obtained by following equation ${ }^{(14)}(10)$.

$$
\lambda_{i}=\lim _{n \rightarrow \infty} \frac{1}{n \Delta \tau} \sum_{j=1}^{n} \ln \left\|\varphi_{i}(j \Delta \tau)\right\|
$$




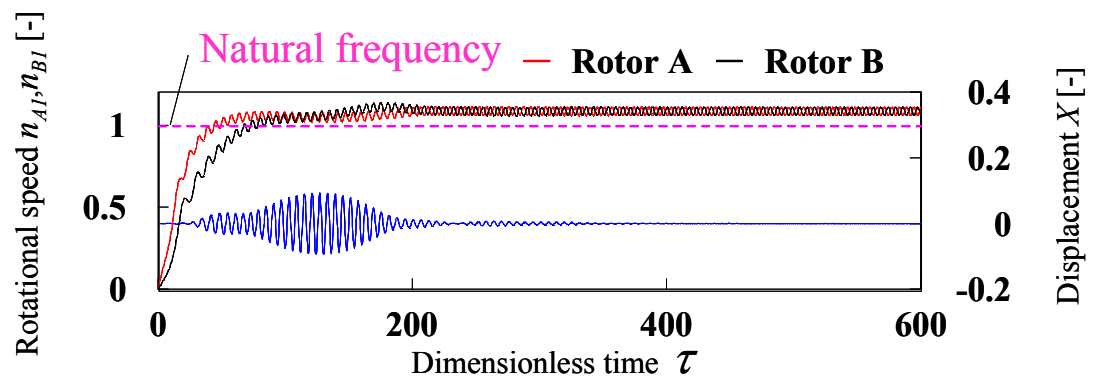

(a) Rotational speeds and displacement of the base plate

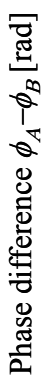

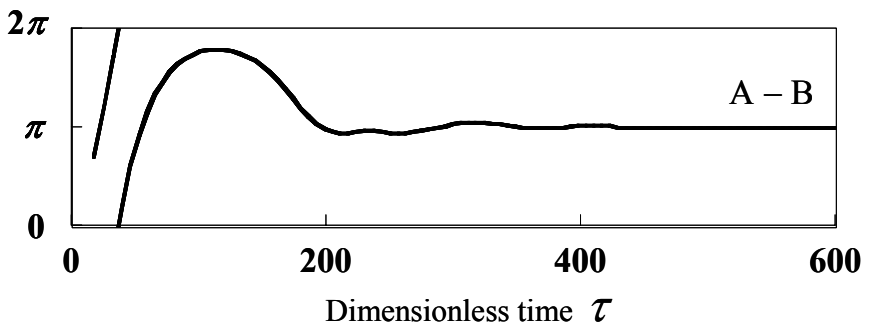

(b) Phase difference $\phi_{A}-\phi_{B}$

Fig. 3 Time histories of the system $(\gamma \eta=0.0078)$

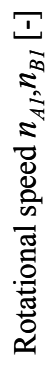

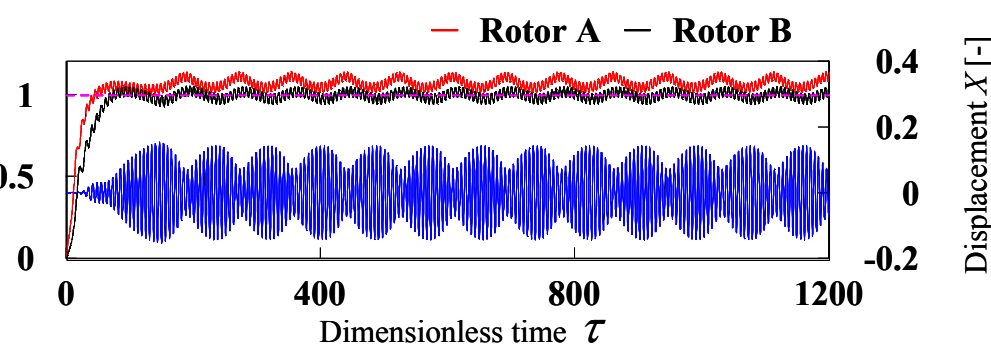

(a) Rotational speeds and displacement of the base plate

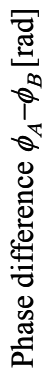

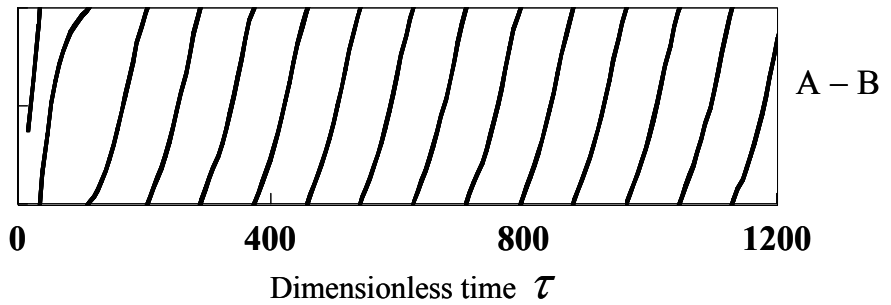

(b) Phase difference $\phi_{A}-\phi_{B}$

Fig. 4 Time histories of the system $(\gamma \eta=0.0085)$

\section{Calculated results and discussion}

We examine the effect of unbalance $\gamma \eta$ on the behavior of the system. Unbalance $\gamma \eta$ are varied from 0.0075 to 0.01 . We set nominal rotational speeds $n_{A 0}$, $n_{B 0}=1.084$. Critical speed is expressed 1.0. When the base plate is excited by rotors, 

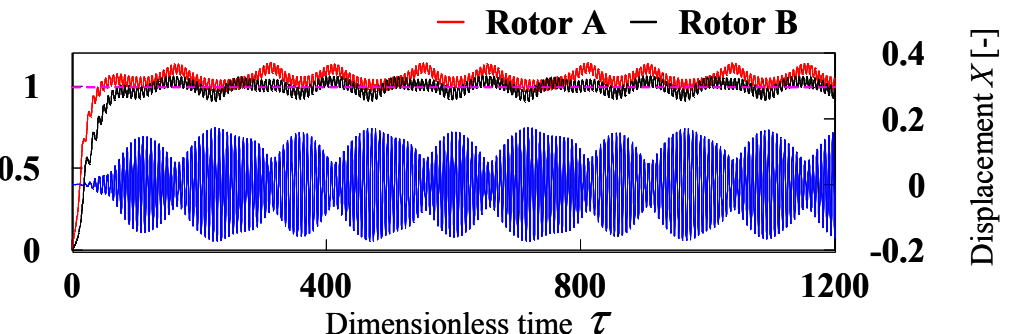

(a) Rotational speeds and displacement of the base plate
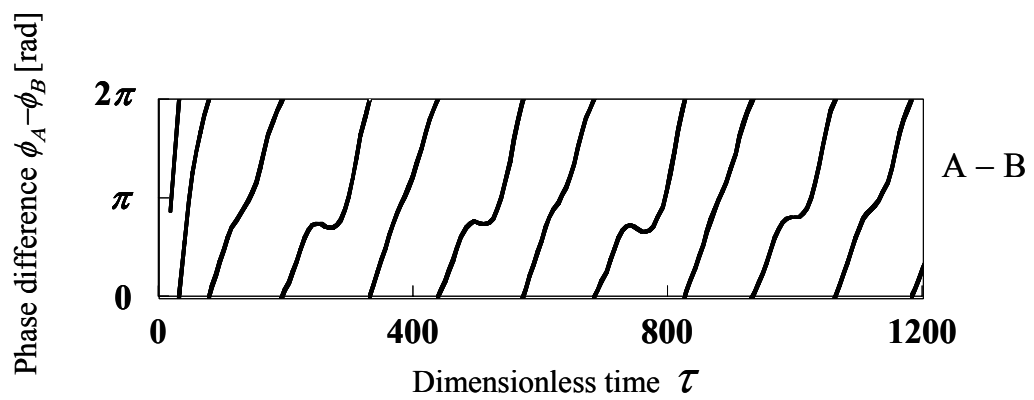

(b) Phase difference $\phi_{A}-\phi_{B}$

Fig. 5 Time histories of the system $(\gamma \eta=0.0094)$

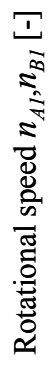

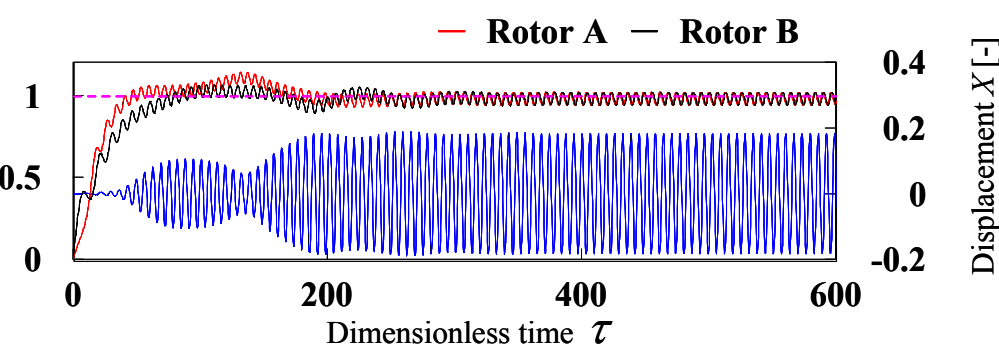

(a) Rotational speeds and displacement of the base plate

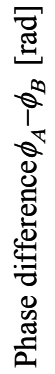

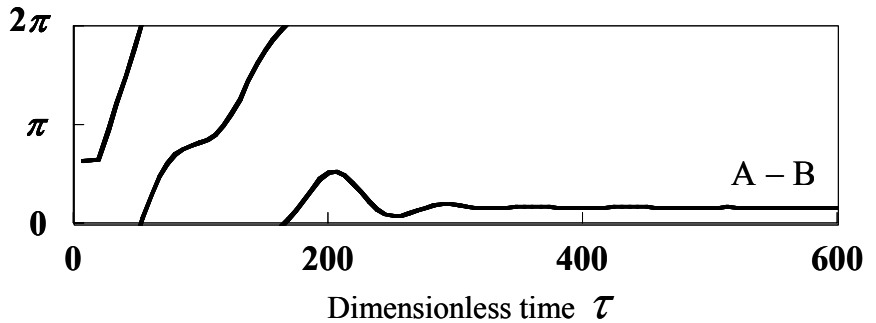

(b) Phase difference $\phi_{A}-\phi_{B}$

Fig. 6 Time histories of the system $(\gamma \eta=0.01)$

the actual rotational speed $n_{i 1}$ of the rotor is influenced by the vibration of the base plate and actual rotational speeds $n_{i 1}$ become less than nominal rotational speeds $n_{i 0}$.

Figure 3 shows calculated results when the rotational speed $n_{i 1}$ passed through the critical speed. Two rotors immediately exceeded the critical speed. The displacement of the base plate also shown in Fig. 3(a). After passing through the critical speed, the amplitude of the base plate became maximum. The free vibration of the system gradually 


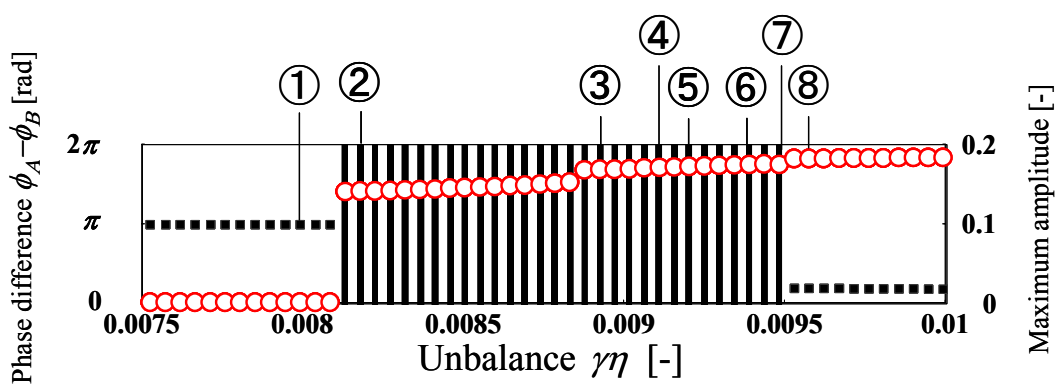

:Phase difference of rotors $A-B$ as $X=0$ and $X^{(1)}>0$ $\bigcirc$ :Maximum amplitude of the base plate

(a) Effect of unbalance
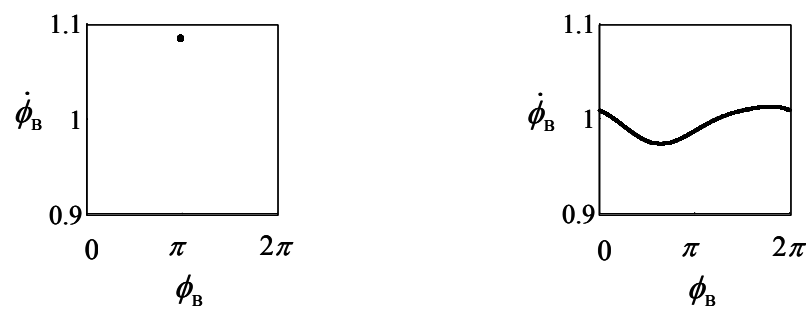

(1) $\gamma \eta=0.0080$

(2) $\gamma \eta=0.0082$
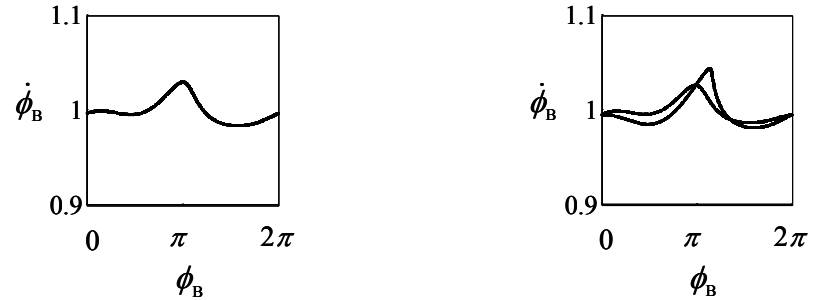

(3) $\gamma \eta=0.0089$

(4) $\gamma \eta=0.0091$
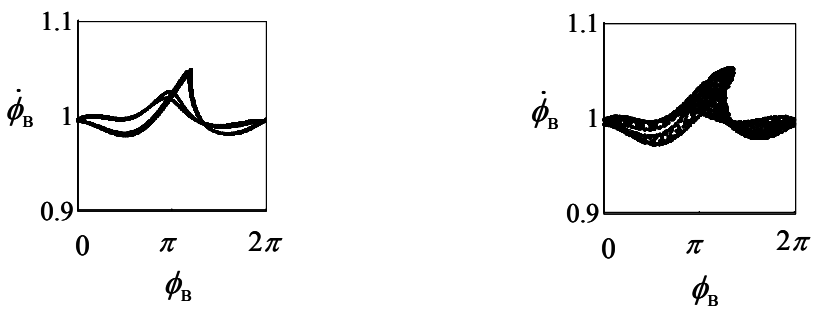

(5) $\gamma \eta=0.0092$

(6) $\gamma \eta=0.0094$
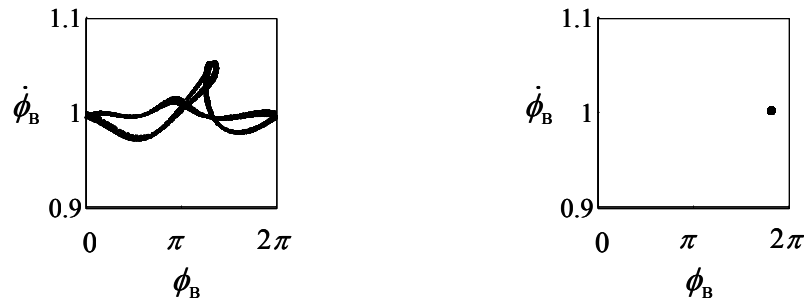

(7) $\gamma \eta=0.0095$

(8) $\gamma \eta=0.0096$

(b) Poincare map of rotor $\mathrm{B}$ as $\phi_{A}=0$

Fig.7 Characteristics of vibration and Poincare map 


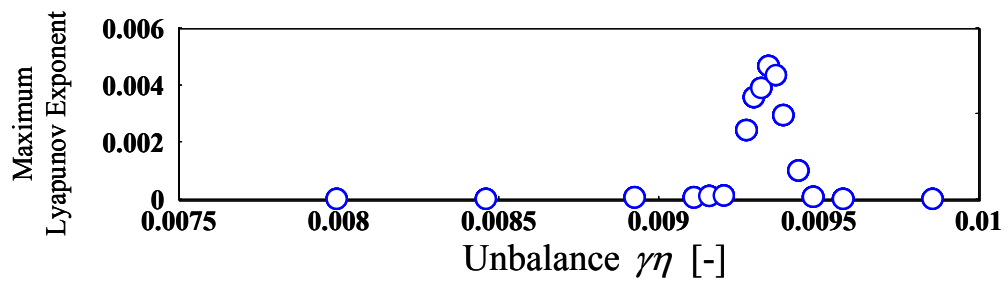

Fig.8 Maximum Lyapunov exponents $\lambda_{i}$

attenuated after reaching the maximum amplitude.

Figure 3(b) shows the phase difference between the rotors, expressed by the angular differences $\phi_{A}-\phi_{B}$. With the attenuation of the free vibration, the phase differences became close to 180 degrees. Under this condition, the rotational speeds are synchronized and unbalanced forces of rotors cancelled each other out. We call this "out-of-phase synchronization". In this case, rotors rotate at more than critical speed stably.

Figure 4 and 5 show the calculated results when unbalance $\gamma \eta$ is bigger than that of Fig. 3. Two rotational speeds did not become identical speed and the maximum amplitudes become very large vibration. Because the slope of torque-speed characteristic curve $\mathrm{B}$ is smaller than that of $\mathrm{A}$ as shown in Fig. 2, actual rotational speed $n_{B 1}$ is captured at critical speed. The phase difference increases as shown in Fig. 4(b) and Fig. 5(b). We call these "non-synchronization". Rotor B is captured at critical speed, whereas rotor A rotates at more than critical speed. This is a reason that non-synchronization occurred. We observed that vibration of the base plate in Fig. 5 is more irregular than that in Fig. 4, and phase difference increase slowly, when phase difference stays near 180 degree as shown in Fig.5(b).

Figure 6 shows the calculated result when the rotational speed did not exceed the critical speed. In this case, unbalance, $\gamma \eta=0.01$, is bigger than that of Fig. 4 and 5. Because the phase of rotors become in phase, the base plate greatly vibrates. We call this "in-phase synchronization". Because both actual rotational speeds $n_{A 1}$ and $n_{B 1}$ are captured at critical speed, in-phase synchronization occurs and base plate is excited violently.

Figure 7(a) shows the phase difference $\phi_{A}-\phi_{B}$ and maximum amplitude to unbalance $\gamma \eta$. Because the out-of-phase synchronization occurs, the maximum amplitude is small as $\gamma \eta$ less than 0.0081 . When the unbalance $\gamma \eta$ is from 0.0082 to 0.0095 , non-synchronization occurs and the amplitude becomes large.

When $\gamma \eta$ is more than 0.0096 , in-phase synchronization occurs and the amplitude becomes large. According to these results, large vibration will occur by increasing of unbalance, even if rotors rotate above the critical speed.

Figure 7(b) shows phase plane (angular velocities vs. angular displacements ) of rotor B as angular displacement of rotor $\mathrm{A} \phi_{A}=0$. According to this result, we observe that the locus of rotor B is complicated from $\gamma \eta=0.0091$ to 0.0095 .

Next we investigated the non-synchronization by Lyapunov exponents. Here, we kept nominal rotational speed $n_{A 0}, n_{B 0}=1.084$. Figure 8 shows maximum Lyapunov exponents to unbalance. The maximum Lyapnov exponents become more than 0 , as the unbalance $\gamma \eta=0.0092 \sim 0.0094$. We can think that vibration of the system as shown in Fig. 5 is chaos and that as shown in Fig. 4 is quasi-periodic vibration. In this analytical model, the unbalance of rotor A and rotor B are almost the same. But torque - speed characteristics are a little different between rotor A and rotor B. We think that is a reason why the chaos occurs in our numerical calculation. So there is a possibility that the chaos depends on the condition of the deference of torque-speed characteristics. 


\section{Conclusions}

We investigate effect of the rotor unbalance on the vibration of the structure which is excited by two rigid rotors numerically. The following conclusions are obtained.

When the unbalanced masses are big, in-phase synchronization occurs. And when the unbalanced mass are small, out-of-phase synchronization occurs. In the vicinity of a border of the two phenomena, unsteady vibration observed. There are two types of non-synchronization, which are quasi-periodic vibration or chaos.

\section{References}

(1) Lewis, F. M., Vibration During Acceleration Through a Critical Speed, Transactions of the ASME, Journal of Applied Mechanics, Vol. 54, (1932), pp.253-261.

(2) Yanabe, S., Vibration of a Shaft Passing Through a Critical Speed (in Japanese), Transactions of the Japan Society of Mechanical Engineers, Series C, Vol.45, No.398 (1979), pp.1082-1091.

(3) Matsuura, K., Dynamics of Rotating Machine Passing Through a Critical Speed (in Japanese), Transactions of the Japan Society of Mechanical Engineers, Series C, Vol.45, No.397 (1979), pp.993-1001.

(4) Ishida, Y., Ikeda, T. and Yamamoto, T., Transient Vibration of a Rotating Shaft with Nonlinear Spring Characteristics During Acceleration Through a Major Critical Speed, Transactions of the Japan Society of Mechanical Engineers, Series C, Vol.52, No.478(1986), 1764-1771.

(5) Iwatsubo, T., Kanki, H. and Kawai, R., Vibration of Asymmetric Rotor Through Critical Speed with Limited Power Supply, Journal Mechanical Engineering Science. Vol.14, No.3 (1972), pp.184-194.

(6) Dornig, A., Transients in Simple Undamped Oscillators Under Inertial Disturbances, Transactions of the ASME, Journal of Applied Mechanics, (1959), pp.217-223.

(7) Ellyin, F. and Wolanski, Z., Transactions of the ASME, Journal of Applied Mechanics, Vol. 53, (1986), pp.417-423.

(8) Dimentberg, M. F., Mcgovern, L., Norton, R. L., Chapdelaine, J. and Harrison R., Dynamics of an Unbalanced Shaft Interacting with a Limited Power Supply, Nonlinear Dynamics, Vol.13, (1997), pp.171-187.

(9) Blekhman, I. I., Synchronization in Science and Technology, (1988), pp.29-36, ASME Press.

(10) Blekhman, I. I., Vibrational Mechanics, (2000), pp.175-196, World Scientific.

(11) Inoue, J. and Miyaura, S., A Study on Interaction Between Power Supply and Machine (in Japanese), Transactions of the Japan Society of Mechanical Engineers, Series C, Vol. 49, No.437 (1983), pp.39-43.

(12) Paz, M. and Cole, J. D., Self-Synchronization of Two Unbalanced Rotors, Transactions of the ASME, Journal of Vibration and Acoustics, Vol. 114, No. 1 (1992), pp.37-41.

(13) Nagamine, T., Sato, Y. and Kawase, K., Vibration Induced by Unbalanced Rigid Rotors Running Through a Critical Speed, Transactions of the Japan Society of Mechanical Engineers, Series C, Vol. 71, No.708 (2005), pp.2439-2446.

(14) For example, Takayasu, H., Science of fractal(in Japanese), (1987), p.85, Asakura Publishing Co. Ltd.. 